\title{
ESTUDO DA SEDIMENTAÇÃO DE BARITA EM FLUIDOS DE PERFURAÇÃO DE POÇOS DE PETRÓLEO
}

\author{
J. M. RIBEIRO Jr. ${ }^{*}$, C. M. SCHEID ${ }^{2}$, L. A. CALÇADA², L. A. C. MELEIRO² \\ ${ }^{1}$ Universidade Federal Rural do Rio de Janeiro, Programa de Pós-Graduação em Engenharia \\ Química \\ ${ }^{2}$ Universidade Federal Rural do Rio de Janeiro, Departamento de Engenharia Química \\ *e-mail: jmessiasrjr@gmail.com
}

\begin{abstract}
RESUMO
A sedimentação de barita é um fenômeno natural que se torna indesejável para a indústria petrolífera devido a vários problemas que ocorrem durante e após a perfuração do poço. O aumento da viscosidade e da pressão do fluido, proporcional ao acúmulo das partículas sólidas de barita, ocasionam dificuldades na perfuração e podem acarretar fraturas na parede do poço. Com o objetivo de predizer o comportamento da sedimentação de barita no fluido de perfuração a base de água, foram feitas várias simulações computacionais através da solução de um sistema de equações diferenciais parciais que descrevem o balanço de massa e das forças que atuam na sedimentação. Foram registradas a velocidade com que as partículas de sólidos sedimentam e a variação da concentração volumétrica de barita ao longo do tempo em vários pontos do poço. Além disso, foram consideradas várias concentrações volumétricas de barita no fluido de perfuração. O sistema de equações diferenciais parciais foi resolvido pelo método das linhas, onde as derivadas espaciais foram discretizadas pelo método das diferenças finitas e o sistema resultante de equações diferenciais ordinárias foi integrado no tempo com a subrotina de domínio público DLSODE, no ambiente de programação FORTRAN. Após essas simulações, foi possível identificar a formação da zona de menor concentração na parte superior do fluido, assim como a zona de compactação na parte inferior da suspensão.
\end{abstract}

\section{INTRODUÇÃO}

Os fluidos de perfuração são utilizados no processo de abertura de poços com o intuito de retirar os fragmentos de rocha abaixo da broca de perfuração ao carreá-los até a superfície, exercer pressão hidrostática contra a parede do poço a fim de prevenir a invasão de fluido de formação no poço, manter o poço recém perfurado aberto até a cimentação do mesmo e para resfriar e lubrificar a linha e a broca de perfuração (BOURGOYNE et al., 1991).

A sedimentação de materiais densos suspensos nos fluidos de perfuração podem ocasionar vários problemas durante a perfuração e conclusão do poço (OMLAND et al., 2007). A sedimentação geralmente começa a uma taxa constante e as partículas são continuamente depositadas no fundo, formando um leito de estrutura solta que gradualmente se consolida como resultado do peso dos sólidos acumulados. O líquido vai sendo redirecionado para o topo através dos espaços dos poros no leito compactado (RICHARDSON e ZAKI, 1954). A formação dessa zona de material compactado e sua subsequente recirculação podem gerar severos problemas operacionais, como dificuldade do controle do poço, perda de circulação, 
instabilidades e entupimentos (ZAMORA e BELL, 2004).

Vários autores investigaram o processo de sedimentação de barita, dentre os quais se destacam os trabalhos de Nguyen, Miska e Takach (2009), que averiguaram a sedimentação dinâmica da barita com escoamento axial de líquido e fluxo axial e radial de sólidos em um tubo cilíndrico inclinado utilizando um fluido de perfuração à base óleo; e de Hashemian et al. (2014), que avaliaram a sedimentação de barita em um anular disposto horizontalmente com escoamento axial de líquido e fluxo bidimensional de sólidos numa suspensão à base óleo.

\subsection{Objetivo}

O presente trabalho tem como principal objetivo o estudo da sedimentação unidirecional de uma suspensão de barita em um fluido de perfuração a base de água na região anular de um poço vertical. Através da solução de um sistema de equações diferenciais parciais que descrevem o balanço de massa e das forças que atuam na sedimentação, foi possível calcular a velocidade com que as partículas de sólidos sedimentam e a variação da concentração volumétrica de barita ao longo do tempo e ao longo do poço. Além disso, foi considerado o efeito da concentração volumétrica de barita no fluido de perfuração.

\section{MATERIAIS E MÉTODOS}

As simulações foram feitas através do uso do método das linhas, com a discretização das derivadas espaciais pelo método das diferenças finitas e o sistema resultante de equações diferenciais ordinárias foi integrado com o integrador DLSODE, no ambiente de programação FORTRAN.

\subsection{Modelo Matemático do Processo}

As equações utilizadas para as simulações consistem na equação da continuidade, representada pela Equação 1, e na equação do movimento, representada pela Equação 2 (SHOOK e ROCO, 1991).

$$
\begin{aligned}
& \frac{\partial\left(c \rho_{s}\right)}{\partial t}+\frac{\partial\left(c \rho_{s} v_{s}\right)}{\partial y}=0 \\
& \rho_{s}\left(\frac{\partial\left(c v_{s}\right)}{\partial t}+v_{s} \frac{\partial\left(c v_{s}\right)}{\partial y}\right)=-\frac{\partial(c P)}{\partial y}+c \rho_{s} g \\
& -c\left(f_{s L}+f_{s s}+f_{s w}\right)
\end{aligned}
$$

Onde a concentração de sólidos no instante inicial é homogênea e a velocidade inicial dos sólidos é obtida através do cálculo da velocidade terminal de Stokes, representada pela equação 3 .

$$
\begin{aligned}
& t=0 \rightarrow c_{0}, \forall y \\
& t=0 \rightarrow v_{0}=v_{\infty}=\frac{g\left(\rho_{s}-\rho_{L}\right) d^{2}}{18 \mu_{L}}, \forall y
\end{aligned}
$$

Nesse estudo, consideram-se os sólidos e o fluido incompressíveis, o gradiente de pressão e a velocidade de líquido nulos, e desprezam-se os efeitos de parede, assim como o termo da aceleração convectiva. A Equação 4 representa a força de interação entre fluido e partícula em um fluxo unidimensional e a Equação 5 descreve a força de interação entre as partículas sólidas, cujo parâmetro $A$ é apresentado na Tabela 1 .

$$
\begin{aligned}
& f_{s L}=\left(\frac{3 C_{D} \rho_{L} v_{s}^{2}}{4 D_{P}(1-c)^{1,7}}\right) \\
& f_{s s}=A c^{2}
\end{aligned}
$$

A substituição das considerações feitas para este trabalho na Equação 1 e na Equação 2 fornecem o sistema de equações diferenciais parciais representado nas Equações 6 e 7, que descrevem o problema da sedimentação de barita em um poço de perfuração. 


$$
\begin{aligned}
& \frac{\partial v_{s}}{\partial t}=g-\frac{f_{s L}}{\rho_{s}}-f_{s s} \\
& \frac{\partial c}{\partial t}=-c \frac{\partial v_{s}}{\partial y}-v_{s} \frac{\partial c}{\partial y}
\end{aligned}
$$

A Equação 8 fornece o coeficiente de arraste para o regime laminar e a Equação 9 representa o número de Reynolds calculado em função da velocidade dos sólidos e da concentração volumétrica de sólidos em um dado ponto.

$$
\begin{aligned}
& C_{D}=\frac{24}{R e} \\
& R e=\frac{D_{P} v_{s} \rho_{L}(1-c)}{\mu_{L}}
\end{aligned}
$$

Tabela 1 - Valores constantes utilizados neste trabalho.

\begin{tabular}{cc}
\hline Variável & Valor \\
\hline$\rho_{s}$ & $3942,29 \mathrm{~kg} / \mathrm{m}^{3}$ \\
$\rho_{L}$ & $1000 \mathrm{~kg} / \mathrm{m}^{3}$ \\
$\mu_{L}$ & $0,001 \mathrm{~kg} /(\mathrm{m} . \mathrm{s})$ \\
$\mathrm{D}_{\mathrm{P}}$ & $0,00002286 \mathrm{~m}$ \\
$\mathrm{~A}$ & $10\left(\mathrm{~m}^{4} / \mathrm{s}^{2}\right)$
\end{tabular}

\subsection{Discretização do Modelo Matemático}

Através da utilização do método das diferenças finitas para a discretização das derivadas espaciais na Equação 7, obtém-se a Equação 10, que juntamente com a Equação 6 geram um sistema de equações diferenciais ordinárias.

$$
\frac{\partial c}{\partial t}=-c \frac{v(i+1)-v(i)}{\Delta y}-v_{s} \frac{c(i+1)-c(i)}{\Delta y}
$$

As condições iniciais para a simulação da sedimentação de barita consistem na imposição da velocidade terminal das partículas no início do processo e da homogeneidade da concentração volumétrica de sólidos no instante inicial.

Figura 1 - Representação dos pontos da malha unidimensional para o problema da sedimentação de barita em um fluido de perfuração.

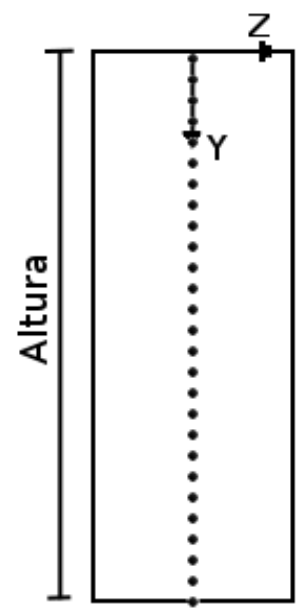

Em cada simulação, a altura do poço foi dividida em 101 pontos com intervalo constante e a integração do sistema de equações diferenciais no tempo foi feito com intervalo de 1 dia.

\section{RESULTADOS E DISCUSSÃO}

Várias simulações foram realizadas utilizando diferentes tempos de simulação, concentrações iniciais de sólido e profundidades do poço de perfuração. Os resultados dessas simulações são apresentados a seguir através de gráficos que relacionam a altura do poço e a concentração volumétrica de sólidos.

Conforme ilustrado na Figura 2, a variação da concentração volumétrica após 1 ano em um poço de 10 metros de profundidade está estabilizado, devido a sedimentação total de sólidos no fundo do poço e a formação da zona de clarificado no topo. 
Figura 2 - Valores obtidos para fluidos com diferentes concentrações de sólidos após um período de 1 ano em um poço com altura de 10 metros.

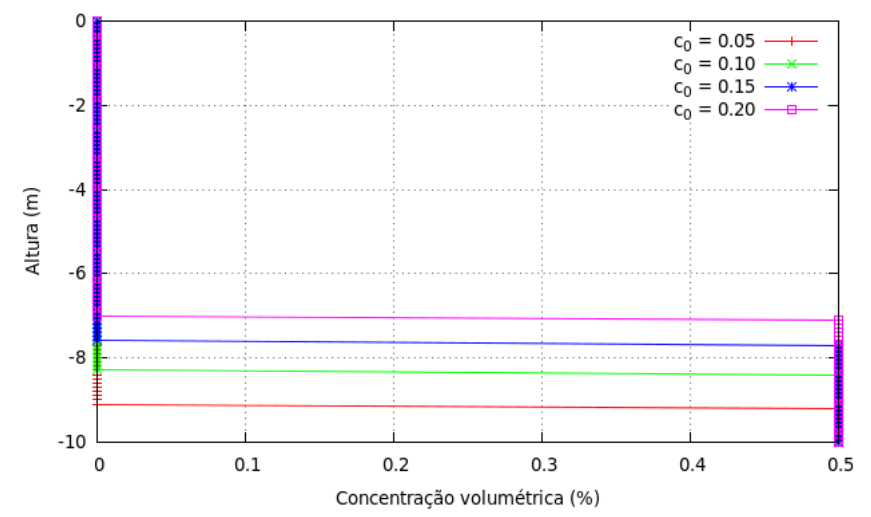

É possível verificar alguma semelhança entre o modelo empírico de Kynch (1952) e os resultados obtidos através de simulação computacional conforme a Figura 3 e a Figura 4.

Figura 3 - Modelo de Kynch (1952) que demonstra a variação da interface entre a zona clarificada e as zonas de sedimentação até a formação total da zona compactada.

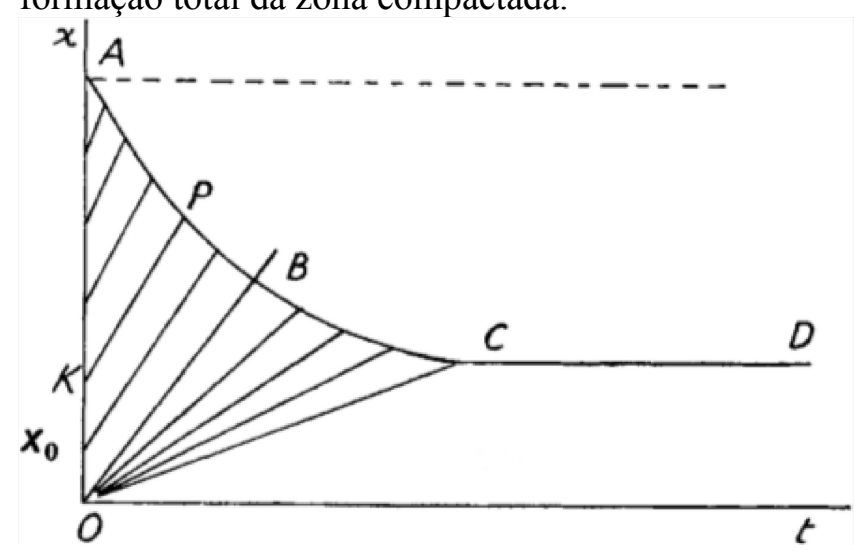

Figura 4 - Valores obtidos para a superfície entre a zona clarificada e a zona de sedimentação com diferentes concentrações de sólidos após um período de 10 anos em um poço com 10 metros de altura.

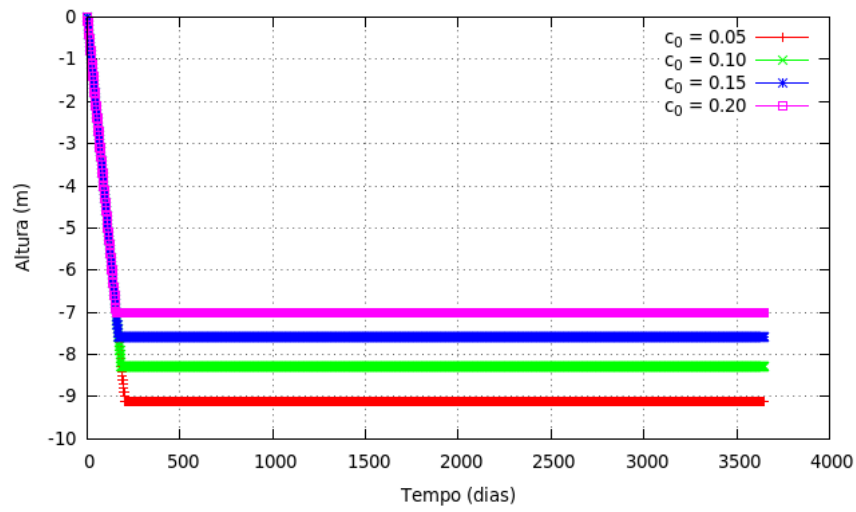

Em um poço com profundidade maior do que na simulação anterior, o gradiente de concentração volumétrico ainda não se estabilizou em 1 ano, mas já é possível perceber a formação da zona de clarificado e a zona de compactação, como pode ser visto na Figura 5 para um poço com 100 metros de profundidade.

Figura 5 - Valores obtidos para fluidos com diferentes concentrações de sólidos após um período de 1 ano em um poço com 100 metros de altura.

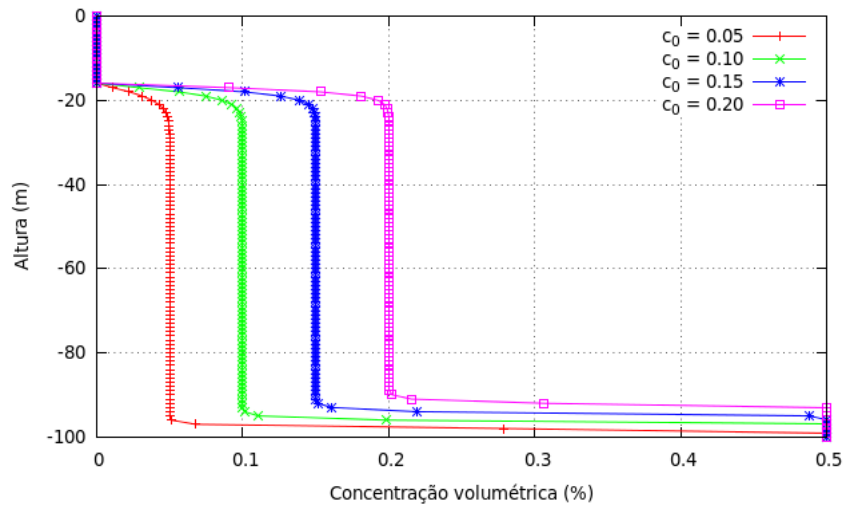

Após um intervalo de tempo ainda maior, verifica-se que ocorre a estabilização da concentração volumétrica. Este efeito pode ser visualizado após 5 anos, onde o perfil de sedimentação já se encontra consolidado. Este comportamento é ilustrado nas Figuras 6 e 7, considerando períodos de 5 e 10 anos, respectivamente. 
Figura 6 - Valores obtidos para fluidos com diferentes concentrações de sólidos após um período de 5 anos em um poço com 100 metros de altura.

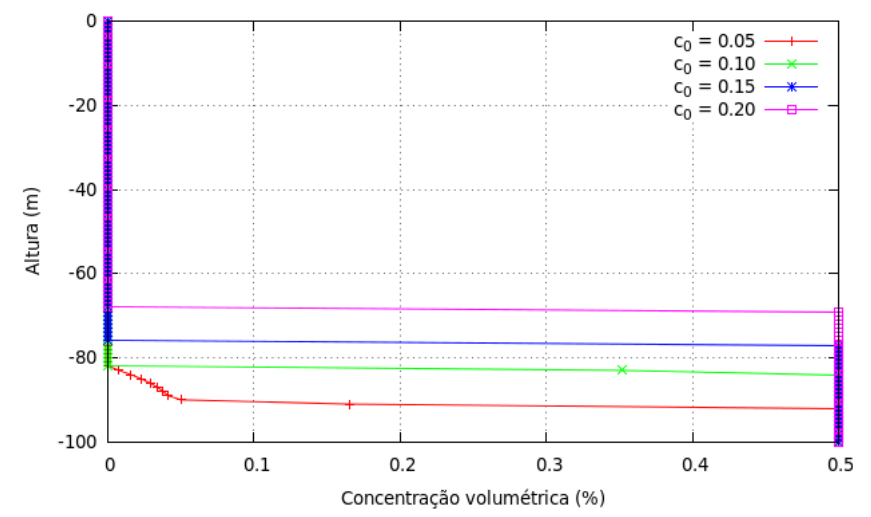

Figura 7 - Valores obtidos para fluidos com diferentes concentrações de sólidos após um período de 10 anos em um poço com 100 metros de altura.

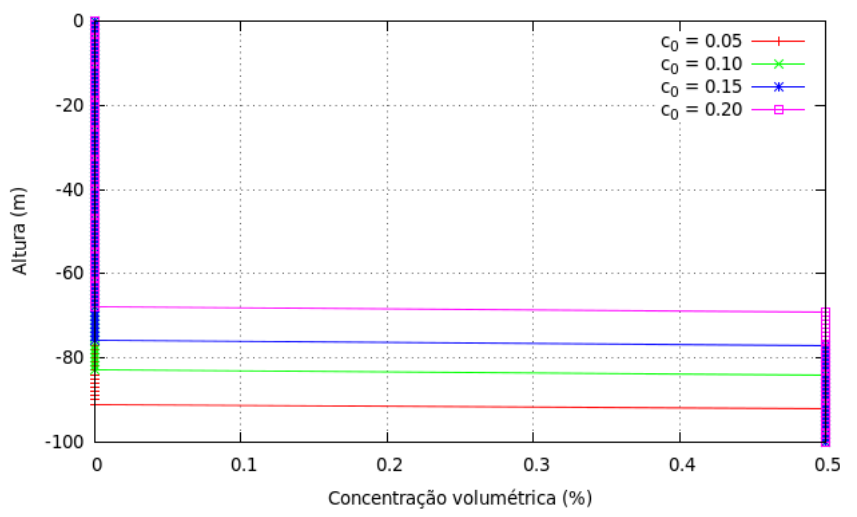

Utilizando o tempo de simulação de 10 anos em um poço de 100 metros de comprimento, é possível constatar a formação do mesmo perfil de sedimentação observado em um poço de 10 metros, com a formação das zonas de sedimentação previstas por Kynch (1952), conforme as Figuras 3 e 8.
Figura 8 - Valores obtidos para a superfície entre a zona clarificada e a zona de sedimentação com diferentes concentrações de sólidos após um período de 10 anos em um poço com 100 metros de altura.

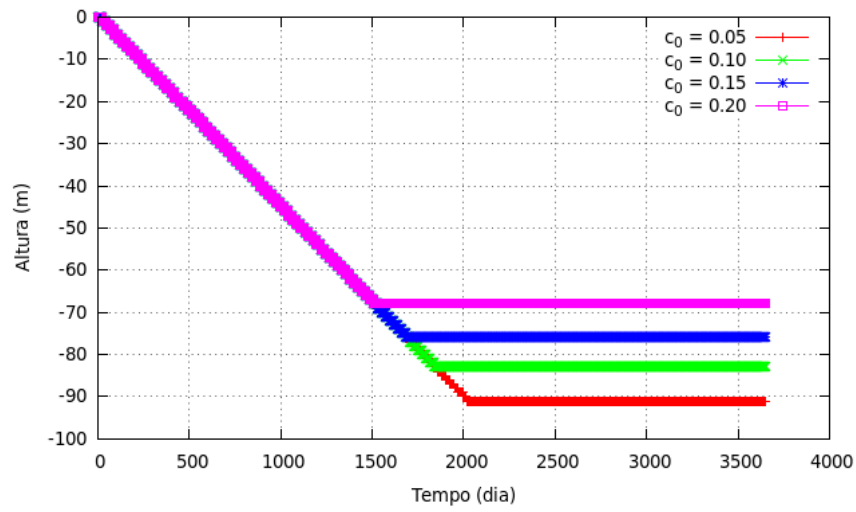

Com o aumento da profundidade do poço para 500 metros, o intervalo de tempo necessário para que ocorra a sedimentação por completo aumenta drasticamente. Para um poço com esta profundidade, o perfil de sedimentação ainda não se encontra estabilizado após 10 anos, mas é possível perceber que existe uma larga zona de fluido clarificado e uma grande zona de sedimentos compactado. Tais características são ilustradas nas Figuras 9 a 11.

Figura 9 - Valores obtidos para fluidos com diferentes concentrações de sólidos após um período de 1 ano em um poço com 500 metros de altura.

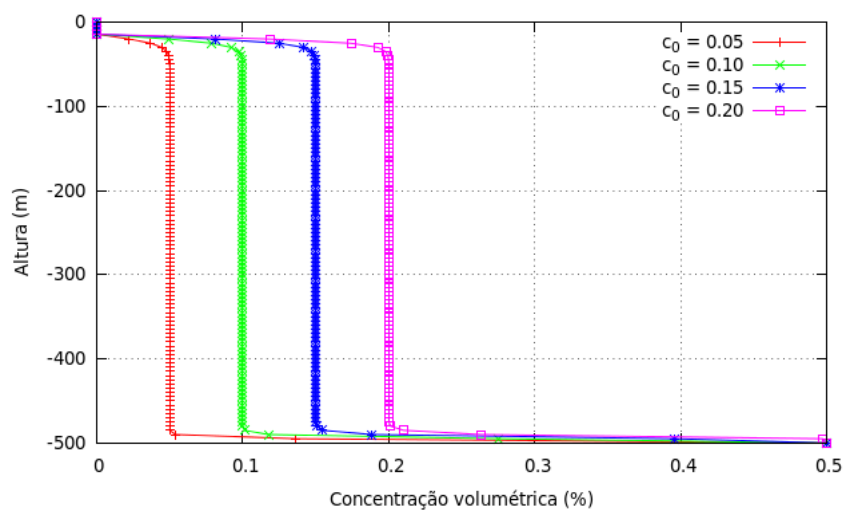


Figura 10 - Valores obtidos para fluidos com diferentes concentrações de sólidos após um período de 5 anos em um poço com 500 metros de altura.

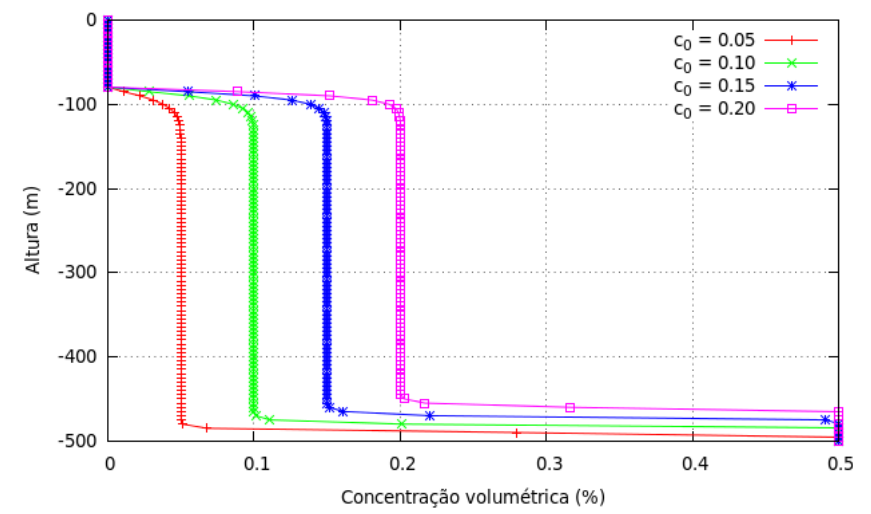

Figura 11 - Valores obtidos para fluidos com diferentes concentrações de sólidos após um período de 10 anos em um poço com 500 metros de altura.

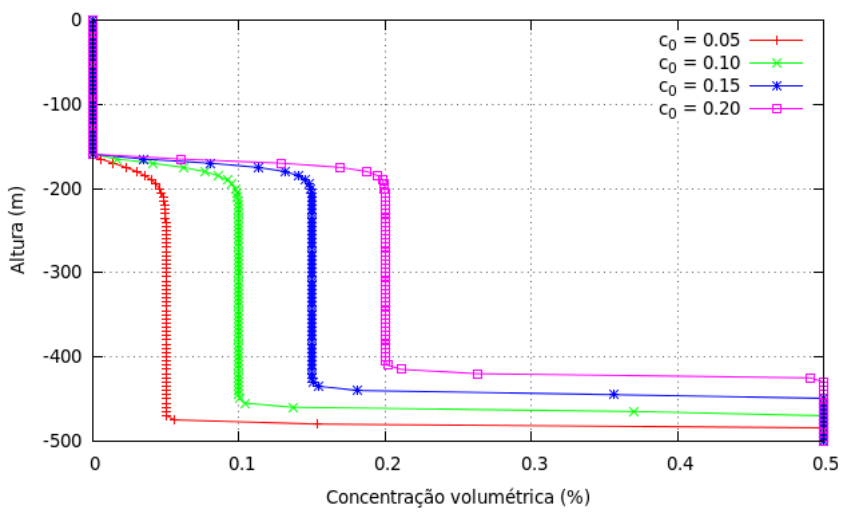

Em um poço com 1000 metros de profundidade, $\mathrm{o}$ gradiente de concentração volumétrico varia lentamente com o tempo, e após um intervalo de tempo de 10 anos ainda existe uma ampla região com concentração volumétrica praticamente constante. Esse comportamento pode ser visualizado nas Figuras 12 a 14.
Figura 12 - Valores obtidos para fluidos com diferentes concentrações de sólidos após um período de 1 ano em um poço com 1000 metros de altura.

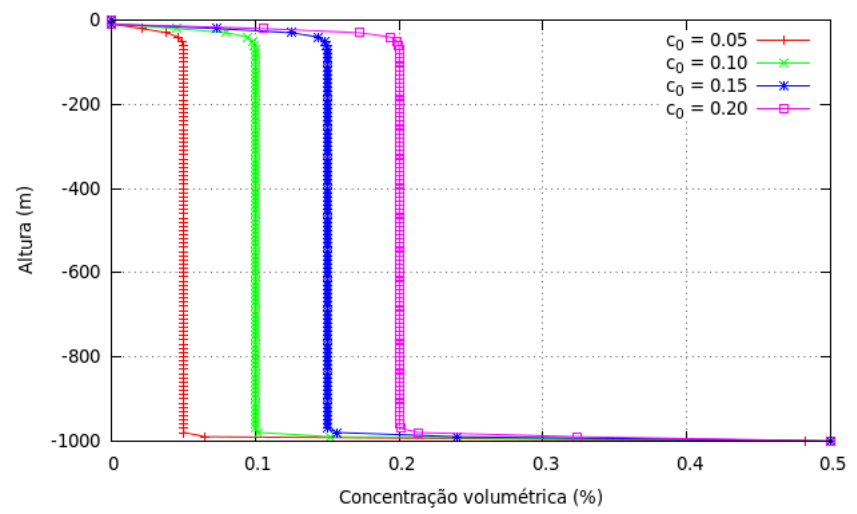

Figura 13 - Valores obtidos para fluidos com diferentes concentrações de sólidos após um período de 5 anos em um poço com 1000 metros de altura.

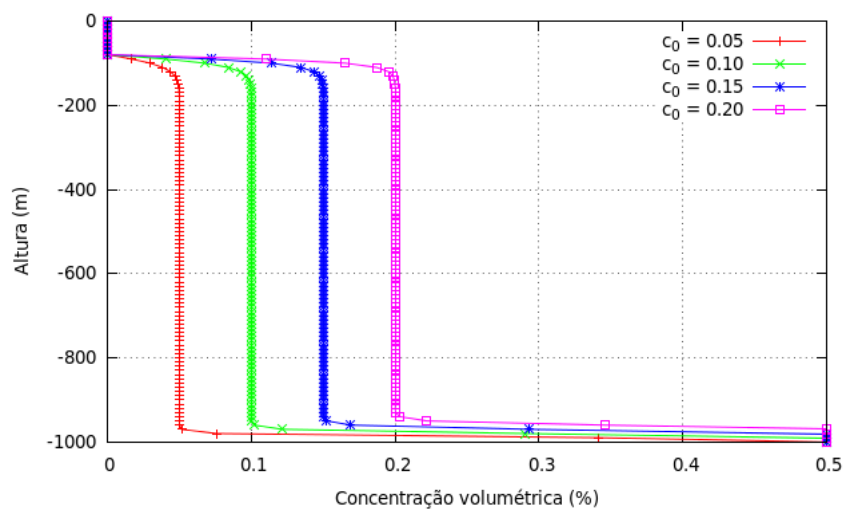

Figura 14 - Valores obtidos para fluidos com diferentes concentrações de sólidos após um período de 10 anos em um poço com 1000 metros de altura.

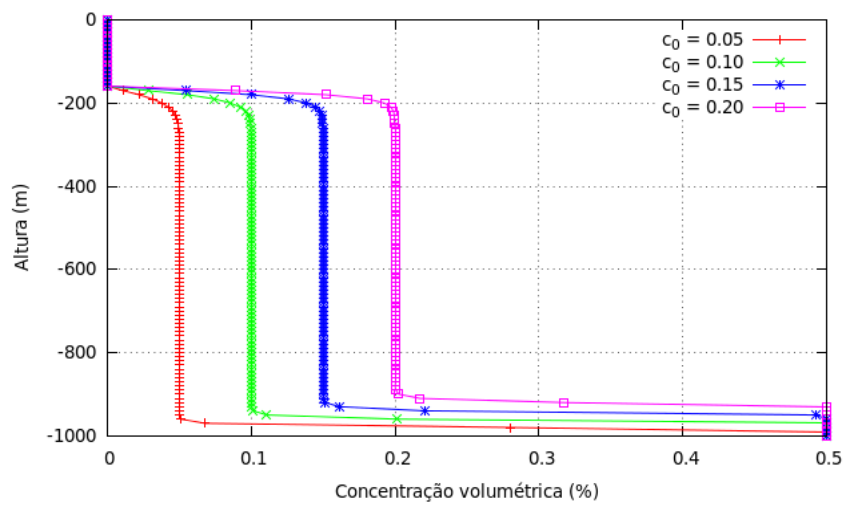




\section{CONCLUSÕES}

Os resultados apresentados neste trabalho ilustram a capacidade do modelo matemático proposto, baseado nas equações de balanço de força e de massa, em descrever o processo de sedimentação da barita em inúmeras situações, de um fluido de perfuração confinado em um poço.

Foi observado que, independentemente do tempo e da altura do poço, os perfis de sedimentação formados se assemelham em todas as simulações, sendo possível distinguir a presença da zona de sedimentação livre, da zona de concentração e da zona de clarificado no fundo do poço.

Os perfis de concentração obtidos são coerentes com o esperado em processos de sedimentação de sólidos, de modo que os resultados fornecidos pelo simulador do processo apresentam bom potencial de uso em processos de perfuração de poços de petróleo, onde a previsão da distribuição de sólidos e, consequentemente, a previsão do perfil de densidade do fluido dentro do poço é de grande interesse operacional.

\section{NOMENCLATURA}

$\mu_{L} \quad$ viscosidade do fluido $[\mathrm{kg} / \mathrm{m} . \mathrm{s}]$

$\rho_{L} \quad$ densidade do fluido $\left[\mathrm{kg} / \mathrm{m}^{3}\right]$

$\rho_{s} \quad$ densidade de sólido $\left[\mathrm{kg} / \mathrm{m}^{3}\right]$

A parâmetro de correlação quadrático $\left[\mathrm{m}^{4} / \mathrm{s}^{2}\right]$

c concentração volumétrica de sólidos

$C_{D} \quad$ coeficiente de arraste

$D_{p} \quad$ diâmetro médio de partícula $[\mu \mathrm{m}]$

$f_{s L}$ força de interação sólidolíquido $\left[\mathrm{kg} \cdot \mathrm{m} / \mathrm{s}^{2}\right]$

$f_{s s}$ força de interação sólidosólido $\left[\mathrm{kg} . \mathrm{m} / \mathrm{s}^{2}\right.$ ]

$f_{s w}$ força de interação sólidoparede $\left[\mathrm{kg} \cdot \mathrm{m} / \mathrm{s}^{2}\right]$ $g \quad$ aceleração da gravidade $\left[\mathrm{m} / \mathrm{s}^{2}\right]$

Re número de Reynolds

$v_{s} \quad$ velocidade de sólido $[\mathrm{m} / \mathrm{s}$ ]

\section{REFERÊNCIAS}

BOURGOYNE, A. T Jr., MILLHEIM, K. K., CHENEVERT, M. E., YOUNG, F. S. Jr. Applied Drilling Engineering. Second Printing SPE Society of Petroleum Engineers. Richardson, Texas, p.41, 1991.

HASHEMIAN Y., MISKA M. Y., MENGJIAO Y., OZBAYYOGLU E., TAKACH N. Numerical Simulation and Experiments of Barite Sag in Horizontal Annulus. American Journal of Numerical Analysis, Vol. 2, No. 1, p.14-19, 2014.

KYNCH, G. J. A Theory of Sedimentation. Transactions of the Faraday Society, v. 48, p. 166-176, 1952.

NGUYEN, T. C., MISKA, M. Y., TAKACH, N. Predicting Dynamic Barite Sag in Newtonian Oil Based Drilling Fluids. SPE Society of Petroleum Engineers. Annual Technical Conference and Exhibition, 4-7 October, New Orleans, Louisiana, 2009.

OMLAND, T. H., SAASEN, A., VAN DER ZWAGG, C., AMUNDSEN, P. A. The Effect of Weighting Material Sag on Drilling Operation Efficiency. SPE Society of Petroleum Engineers. Asia Pacific Oil and Gas Conference and Exhibition, Jacarta, Indonésia, 2007.

RICHARDSON, J. F., ZAKI, W. N. Sedimentation and Fluidization: Part I. Transaction Institution of Chemical Engineers, Vol. 32, 1954.

SHOOK, C. A., ROCO, M. C. Slurry Flow Principles and Practice. ButterworthHeinemann series in chemical engineering, p.29-30, 1991. 
ZAMORA, M., BELL, R. Improved Wellsite Test for Monitoring Barite Sag . AADE American Association of Drilling Engineers, Houston, Texas, 2004.

\section{AGRADECIMENTOS}

Agradeço a Petrobras pelos recursos disponibilizados, sem os quais não seria possível este trabalho. 